\title{
THE DECAY OF THE WALSH COEFFICIENTS OF SMOOTH FUNCTIONS
}

\author{
JOSEF DICK
}

(Received 11 December 2008)

\begin{abstract}
We give upper bounds on the Walsh coefficients of functions for which the derivative of order at least one has bounded variation of fractional order. Further, we also consider the Walsh coefficients of functions in periodic and nonperiodic reproducing kernel Hilbert spaces. A lower bound which shows that our results are best possible is also shown.
\end{abstract}

2000 Mathematics subject classification: primary 42C10.

Keywords and phrases: wavelet, Walsh series, Walsh coefficient, sobolev space, smooth function.

\section{Introduction}

In this paper we analyze the decay of the Walsh coefficients of smooth functions. Walsh functions wal $_{k}:[0,1) \rightarrow\left\{1, \omega_{b}, \ldots, \omega_{b}^{b-1}\right\}$, where $k$ is a nonnegative integer and $\omega_{b}=e^{2 \pi i / b}$, were first introduced in [11] and further early results were obtained in $[2,6]$. See for example [9] for an overview. It is well known that Walsh functions form a complete orthonormal system of $L_{2}([0,1))$, see [2, 7].

In analogy to our aim for Walsh functions, consider Fourier series for a moment: a classical result says that the $k$ th Fourier coefficient of an $r$ times differentiable function decays with order $|k|^{-r}$. An analogous result for the Walsh coefficients of $r$ times differentiable functions has been missing in the literature and is provided here.

The Walsh coefficients of functions which satisfy a Hölder condition were already considered in [6]. Here we consider the decay of the Walsh coefficients of functions which satisfy even stronger smoothness assumptions, that is, have at least one smooth derivative. It has long been known from [6] that the only absolutely continuous functions for which all Walsh coefficients decay faster than $1 / k$ are the constants. Here we refine this result by showing that for $r$ times differentiable functions, the Walsh co-

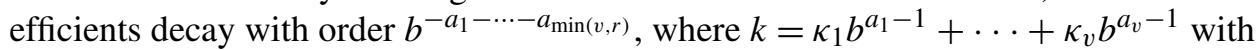
$0<\kappa_{1}, \ldots, \kappa_{v}<b$ and $a_{1}>\cdots>a_{v}>0$. That is, only the coefficients $k$ which have only one nonzero digit in their base $b$ expansion decay with order $1 / k$, the others decay faster. We also prove a lower bound which shows that this result is the best possible.

(C) 2009 Australian Mathematical Publishing Association Inc. 0004-9727/2009 \$16.00 
The question of how the Walsh coefficients of smooth functions decay plays a central role in numerical integration of smooth functions. In [3, 4] this decay was implicitly used to give explicit constructions of quasi-Monte Carlo rules which achieve the optimal rate of convergence for the numerical integration of functions with smoothness $r>1$.

Throughout the paper we use the following notation: we assume that $b \geq 2$ is a natural number, and that $k \in \mathbb{N}$ (where $\mathbb{N}$ denotes the set of natural numbers) has base $b$ expansion $k=\kappa_{1} b^{a_{1}-1}+\cdots+\kappa_{v} b^{a_{v}-1}$, where $v \geq 1,0<\kappa_{1}, \ldots, \kappa_{v}<b$, and $a_{1}>\cdots>a_{v}>0$. For $k=0$ we assume that $v=0$.

Let the real number $x \in[0,1)$ have base $b$ representation $x=x_{1} / b+x_{2} / b^{2}+\cdots$, with $0 \leq x_{i}<b$ and where infinitely many $x_{i}$ are different from $b-1$. For $k \in \mathbb{N}$ we define the $k$ th Walsh function by

$$
\operatorname{wal}_{k}(x)=\omega_{b}^{\kappa_{1} x_{a_{1}}+\cdots+\kappa_{v} x_{a_{v}}},
$$

where $\omega_{b}=e^{2 \pi i / b}$. For $k=0$ we set $\operatorname{wal}_{0}(x)=1$.

For a function $f:[0,1] \rightarrow \mathbb{R}$ we define the $k$ th Walsh coefficient of $f$ by

$$
\hat{f}(k)=\int_{0}^{1} f(x) \overline{\mathrm{wal}_{k}(x)} d x
$$

and we can form the Walsh series

$$
f(x) \sim \sum_{k=0}^{\infty} \hat{f}(k) \mathrm{wal}_{k}(x) .
$$

Among other things, it was shown in [4] that if a function $f:[0,1] \rightarrow \mathbb{R}$ has $r-1$ derivatives for which $f^{(r-1)}$ satisfies a Lipschitz condition, then $|\hat{f}(k)| \leq$ $C_{r} b^{-a_{1}-\cdots-a_{\min (r, v)}}$ for some constant $C_{r}>0$ independent of $f$ and $k$. An explicit constant was also given in [4].

In this paper we improve upon the results in [3] and [4] in several ways. We improve the constant $C_{r}$ mentioned above and also obtain a constant for $r=\infty$ (in [4] we have $C_{r} \rightarrow \infty$ for $r \rightarrow \infty$ ). (In the context of numerical integration this is interesting as we want to know how the integration error depends on the smoothness.) If the function and all of its derivatives are periodic, then the result can be strengthened. This was already used implicitly in [3], but will be shown explicitly here.

We need the following lemma which was first shown in [6] and appeared in many other papers (see, for example, [4] for a more general version). The following notation is used throughout the paper: $k^{\prime}=k-\kappa_{1} b^{a_{1}-1}$ and, hence, $0 \leq k^{\prime}<b^{a_{1}-1}$.

LeMma 1. For $k \in \mathbb{N}$ let $J_{k}(x)=\int_{0}^{x} \overline{\operatorname{wal}_{k}(t)} d t$. Then

$$
\begin{gathered}
J_{k}(x)=b^{-a_{1}}\left(\left(1-\omega_{b}^{-\kappa_{1}}\right)^{-1} \overline{\mathrm{wal}_{k^{\prime}}(x)}+\left(1 / 2+\left(\omega_{b}^{-\kappa_{1}}-1\right)^{-1}\right) \overline{\mathrm{wal}_{k}(x)}\right. \\
\left.+\sum_{c=1}^{\infty} \sum_{\vartheta=1}^{b-1} b^{-c}\left(\omega_{b}^{\vartheta}-1\right)^{-1} \overline{\mathrm{wal}_{\vartheta b^{a_{1}+c-1}+k}(x)}\right) .
\end{gathered}
$$


For $k=0$, that is, $J_{0}(x)=\int_{0}^{x} 1 d t=x$, we have

$$
J_{0}(x)=1 / 2+\sum_{c=1}^{\infty} \sum_{\vartheta=1}^{b-1} b^{-c}\left(\omega_{b}^{\vartheta}-1\right)^{-1} \overline{\mathrm{wal}_{\vartheta b^{c-1}}(x)} .
$$

We also need the following elementary lemma.

LEMMA 2. For any $0<\kappa<b$ we have

$$
\left|1-\omega_{b}^{-\kappa}\right|^{-1} \leq \frac{1}{2 \sin (\pi / b)} \quad \text { and } \quad\left|1 / 2+\left(\omega_{b}^{-\kappa}-1\right)^{-1}\right| \leq \frac{1}{2 \sin (\pi / b)} .
$$

We introduce some further notation which is used throughout the paper: for $v>1$ let $k^{\prime \prime}=k^{\prime}-\kappa_{2} b^{a_{2}-1}$, and hence $0 \leq k^{\prime \prime}<b^{a_{2}-1}$. For $l \in \mathbb{N}$ let $l=\lambda_{1} b^{d_{1}-1}+\cdots+$ $\lambda_{w} b^{d_{w}-1}$, where $w \geq 1,0<\lambda_{1}, \ldots, \lambda_{w}<b$, and $d_{1}>\cdots>d_{w}>0$. Further, let $l^{\prime}=l-\lambda_{1} b^{d_{1}-1}$ and hence $0 \leq l^{\prime}<b^{d_{1}-1}$. For $w>1$ let $l^{\prime \prime}=l^{\prime}-\lambda_{2} b^{d_{2}-1}$, and hence $0 \leq l^{\prime \prime}<b^{d_{2}-1}$.

\section{On the Walsh coefficients of polynomials and power series}

In the following we obtain bounds on the Walsh coefficients of monomials $x^{r}$. Let

$$
\chi_{r, v}\left(a_{1}, \ldots, a_{v} ; \kappa_{1}, \ldots, \kappa_{v}\right)=\int_{0}^{1} x^{r} \overline{\operatorname{wal}_{k}(x)} d x .
$$

For $k=0$ we define $\chi_{r, 0}$, which is given by

$$
\chi_{r, 0}=\int_{0}^{1} x^{r} d x=\frac{1}{r+1} .
$$

We know from [4, Lemma 3.7] that the Walsh coefficients of $x^{r}$ are zero if $v>r$, hence we have $\chi_{r, v}=0$ for $v>r$.

The Walsh series for $x$ is already known from Lemma 1, thus (note that we need to take the complex conjugate of (1) to obtain the Walsh series for $x$ )

$$
\chi_{1,1}\left(a_{1} ; \kappa_{1}\right)=-b^{-a_{1}}\left(1-\omega_{b}^{-\kappa_{1}}\right)^{-1} .
$$

It can be checked that $\left|\chi_{1,1}\right| \leq \frac{1}{2}$. Indeed, we always have

$$
\left|\chi_{r, v}\left(a_{1}, \ldots, a_{v} ; \kappa_{1}, \ldots, \kappa_{v}\right)\right| \leq \int_{0}^{1} x^{r}\left|\overline{\operatorname{wal}_{k}(x)}\right| d x=\int_{0}^{1} x^{r} d x=\frac{1}{r+1}
$$

for all $r, v \geq 0$.

We obtain a recursive formula for the $\chi_{r, v}$ using integration by parts, namely

$$
\int_{0}^{1} x^{r} \overline{\operatorname{wal}_{k}(x)} d x=\left.J_{k}(x) x^{r}\right|_{0} ^{1}-r \int_{0}^{1} x^{r-1} J_{k}(x) d x=-r \int_{0}^{1} x^{r-1} J_{k}(x) d x .
$$


Using Lemma 1 and (3) we obtain for $1 \leq v \leq r$ and $r>1$ that

$$
\begin{aligned}
\chi_{r, v}\left(a_{1}, \ldots, a_{v} ; \kappa_{1}, \ldots, \kappa_{v}\right) \\
=-r b^{-a_{1}}\left(\left(1-\omega_{b}^{-\kappa_{1}}\right)^{-1} \chi_{r-1, v-1}\left(a_{2}, \ldots, a_{v} ; \kappa_{2}, \ldots, \kappa_{v}\right)\right. \\
\quad+\left(1 / 2+\left(\omega_{b}^{-\kappa_{1}}-1\right)^{-1}\right) \chi_{r-1, v}\left(a_{1}, \ldots, a_{v} ; \kappa_{1}, \ldots, \kappa_{v}\right) \\
\left.\quad+\sum_{c=1}^{\infty} \sum_{\vartheta=1}^{b-1} b^{-c}\left(\omega_{b}^{\vartheta}-1\right)^{-1} \chi_{r-1, v+1}\left(a_{1}+c, a_{1}, \ldots, a_{v} ; \vartheta, \kappa_{1}, \ldots, \kappa_{v}\right)\right) .
\end{aligned}
$$

From (4) we can obtain

$$
\chi_{r, r}\left(a_{1}, \ldots, a_{r} ; \kappa_{1}, \ldots, \kappa_{r}\right)=(-1)^{r} r ! b^{-a_{1}-\cdots-a_{r}} \prod_{w=1}^{r}\left(1-\omega_{b}^{-\kappa_{w}}\right)^{-1}
$$

and, with a bit more effort,

$$
\begin{aligned}
\chi_{r, r-1}\left(a_{1}, \ldots, a_{r-1} ; \kappa_{1}, \ldots, \kappa_{r-1}\right) \\
=(-1)^{r} r ! b^{-a_{1}-\cdots-a_{r-1}} \prod_{w=1}^{r-1}\left(1-\omega_{b}^{-\kappa_{w}}\right)^{-1} \\
\quad \times\left(-1 / 2+\sum_{w=1}^{r-1}\left(1 / 2+\left(\omega_{b}^{-\kappa_{w}}-1\right)^{-1}\right) b^{-a_{w}}\right),
\end{aligned}
$$

for all $r \geq 1$.

In principle we can obtain all values of $\chi_{r, v}$ recursively using (4). We have already calculated $\chi_{r, v}$ for $v=r, r-1$ and we could continue doing so for $v=r-2, \ldots, 1$. However, the formulae become increasingly complex, so we only prove a bound on them.

For any $r \geq 0$ and a nonnegative integer $k$ we define

$$
\mu_{r}(k)= \begin{cases}0 & \text { for } r=0, k \geq 0, \\ 0 & \text { for } k=0, r \geq 0, \\ a_{1}+\cdots+a_{v} & \text { for } 1 \leq v \leq r \\ a_{1}+\cdots+a_{r} & \text { for } v>r\end{cases}
$$

LEMMA 3. For $1 \leq r<v$ we have $\chi_{r, v}=0$ and for any $1 \leq v \leq r$ we have

$$
\begin{aligned}
& \left|\chi_{r, v}\left(a_{1}, \ldots, a_{v} ; \kappa_{1}, \ldots, \kappa_{v}\right)\right| \\
& \quad \leq \min _{0 \leq u \leq v} b^{-\mu_{u}(k)} \frac{r !}{(r-u+1) !} \frac{3^{\min (1, u)}}{(2 \sin (\pi / b))^{u}}\left(1+\frac{1}{b}+\frac{1}{b(b+1)}\right)^{\max (0, u-1)} .
\end{aligned}
$$

PROOF. The first result was already shown in [4].

For the second result we use induction on $r$. We have already shown the result for $r=v=1$. 
Now assume that

$$
\begin{aligned}
& \left|\chi_{r-1, v}\left(a_{1}, \ldots, a_{v} ; \kappa_{1}, \ldots, \kappa_{v}\right)\right| \\
& \quad \leq \min _{0 \leq u \leq v} b^{-\mu_{u}(k)} \frac{(r-1) !}{(r-u) !} \frac{3^{\min (1, u)}}{(2 \sin (\pi / b))^{u}}\left(1+\frac{1}{b}+\frac{1}{b(b+1)}\right)^{\max (0, u-1)} .
\end{aligned}
$$

We show that the result holds for $r$. We have already shown that $\left|\chi_{r, v}\right| \leq 1 /(r+1)$, which proves the result for $u=0$.

By taking the absolute value of (4) and using the triangular inequality we obtain

$$
\begin{aligned}
& \left|\chi_{r, v}\left(a_{1}, \ldots, a_{v} ; \kappa_{1}, \ldots, \kappa_{v}\right)\right| \\
& \leq r b^{-a_{1}}\left(\left|1-\omega_{b}^{-\kappa_{1}}\right|^{-1}\left|\chi_{r-1, v-1}\left(a_{2}, \ldots, a_{v} ; \kappa_{2}, \ldots, \kappa_{v}\right)\right|\right. \\
& \quad+\left|1 / 2+\left(\omega_{b}^{-\kappa_{1}}-1\right)^{-1}\right|\left|\chi_{r-1, v}\left(a_{1}, \ldots, a_{v} ; \kappa_{1}, \ldots, \kappa_{v}\right)\right| \\
& \quad+\sum_{c=1}^{\infty} \sum_{\vartheta=1}^{b-1} b^{-c}\left|\omega_{b}^{\vartheta}-1\right|^{-1} \\
& \left.\quad \times\left|\chi_{r-1, v+1}\left(a_{1}+c, a_{1}, \ldots, a_{v} ; \vartheta, \kappa_{1}, \ldots, \kappa_{v}\right)\right|\right) .
\end{aligned}
$$

Using Lemma 2, $\left|\chi_{r-1, v}\right| \leq 1 / r$ and $\sum_{c=1}^{\infty} b^{-c}=1 /(b-1)$, we obtain from (5) that

$$
\left|\chi_{r, v}\left(a_{1}, \ldots, a_{v} ; \kappa_{1}, \ldots, \kappa_{v}\right)\right| \leq \frac{3 b^{-a_{1}}}{2 \sin (\pi / b)},
$$

which proves the bound for $u=1$.

To prove the bound for $1<u \leq v$ we proceed in the same manner. Using Lemma 2, and

$$
\begin{aligned}
& \left|\chi_{r-1, v}\left(a_{1}, \ldots, a_{v} ; \kappa_{1}, \ldots, \kappa_{v}\right)\right| \\
& \quad \leq b^{-\mu_{u-1}(k)} \frac{(r-1) !}{(r-u+1) !} \frac{3^{\min (1, u-1)}}{(2 \sin (\pi / b))^{u-1}}\left(1+\frac{1}{b}+\frac{1}{b(b+1)}\right)^{\max (0, u-2)},
\end{aligned}
$$

we obtain

$$
\begin{aligned}
& \left|\chi_{r, v}\left(a_{1}, \ldots, a_{v} ; \kappa_{1}, \ldots, \kappa_{v}\right)\right| \\
& \leq \frac{r b^{-a_{1}}}{2 \sin (\pi / b)}\left(\left|\chi_{r-1, v-1}\left(a_{2}, \ldots, a_{v} ; \kappa_{2}, \ldots, \kappa_{v}\right)\right|\right. \\
& \quad+\left|\chi_{r-1, v}\left(a_{1}, \ldots, a_{v} ; \kappa_{1}, \ldots, \kappa_{v}\right)\right| \\
& \left.\quad+\sum_{c=1}^{\infty} \sum_{\vartheta=1}^{b-1} b^{-c}\left|\chi_{r-1, v+1}\left(a_{1}+c, a_{1}, \ldots, a_{v} ; \vartheta, \kappa_{1}, \ldots, \kappa_{v}\right)\right|\right)
\end{aligned}
$$




$$
\begin{aligned}
& \leq b^{-\mu_{u}(k)} \frac{r !}{(r-u+1) !} \frac{3^{\min (1, u)}}{(2 \sin (\pi / b))^{u}}\left(1+\frac{1}{b}+\frac{1}{b(b+1)}\right)^{\max (0, u-2)} \\
& \quad \times\left(1+b^{a_{2}-a_{1}}+\frac{b^{a_{2}-a_{1}}}{b+1}\right) \\
& \leq b^{-\mu_{u}(k)} \frac{r !}{(r-u+1) !} \frac{3^{\min (1, u)}}{(2 \sin (\pi / b))^{u}}\left(1+\frac{1}{b}+\frac{1}{b(b+1)}\right)^{\max (0, u-1)}
\end{aligned}
$$

as $\sum_{c=1}^{\infty} \sum_{\vartheta=1}^{b-1} b^{-2 c}=1 /(b+1)$ and $a_{1}>a_{2}$. Thus, the result follows.

Let now $f(x)=f_{0}+f_{1} x+f_{2} x^{2}+\cdots$. The $k$ th Walsh coefficient of $f$ is given by

$$
\begin{aligned}
\hat{f}(k) & =\int_{0}^{1} f(x) \overline{\mathrm{wal}_{k}(x)} d x \\
& =\sum_{r=0}^{\infty} f_{r} \int_{0}^{1} x^{r} \overline{\operatorname{wal}_{k}(x)} d x \\
& =\sum_{r=v}^{\infty} f_{r} \chi_{r, v}\left(a_{1}, \ldots, a_{v} ; \kappa_{1}, \ldots, \kappa_{v}\right) .
\end{aligned}
$$

We can estimate the $k$ th Walsh coefficient by

$$
\begin{aligned}
|\hat{f}(k)| & =\left|\sum_{r=v}^{\infty} \chi_{r, v}\left(a_{1}, \ldots, a_{v} ; \kappa_{1}, \ldots, \kappa_{v}\right) f_{r}\right| \\
\leq & \sum_{r=v}^{\infty}\left|\chi_{r, v}\left(a_{1}, \ldots, a_{v} ; \kappa_{1}, \ldots, \kappa_{v}\right)\right|\left|f_{r}\right| \\
\leq & \sum_{r=v}^{\infty}\left|f_{r}\right| \min _{0 \leq u \leq v} b^{-\mu_{u}(k)} \frac{r !}{(r-u+1) !} \frac{3^{\min (1, u)}}{(2 \sin (\pi / b))^{u}} \\
& \times\left(1+\frac{1}{b}+\frac{1}{b(b+1)}\right)^{\max (0, u-1)} \\
\leq & \min _{0 \leq u \leq v} b^{-\mu_{u}(k)} \frac{3^{\min (1, u)}}{(2 \sin (\pi / b))^{u}}\left(1+\frac{1}{b}+\frac{1}{b(b+1)}\right) \\
& \times \sum_{r=v}^{\infty} \frac{r !\left|f_{r}\right|}{(r-u+1) !} .
\end{aligned}
$$

Hence, we have shown the following theorem.

THEOREM 4. Let $f(x)=f_{0}+f_{1} x+f_{2} x^{2}+\cdots$ and let $k \in \mathbb{N}$. Then

$$
\begin{aligned}
|\hat{f}(k)| \leq \min _{0 \leq u \leq v} & b^{-\mu_{u}(k)} \frac{3^{\min (1, u)}}{(2 \sin (\pi / b))^{u}} \\
& \times\left(1+\frac{1}{b}+\frac{1}{b(b+1)}\right)^{\max (0, u-1)} \sum_{r=v}^{\infty} \frac{r !\left|f_{r}\right|}{(r-u+1) !} .
\end{aligned}
$$


REMARK 5. This result cannot be obtained directly from [4], as there the constant for a power series would be infinite.

The bound in the theorem of course only makes sense for $u$ for which $\sum_{r=v}^{\infty}\left(r !\left|f_{r}\right| /(r-u+1) !\right)$ is finite. We give some examples.

- For $f \in C^{\infty}([0,1])$ we have $f^{(r)}(0)=r ! f_{r}$. If $\left|f^{(r)}(0)\right|$ grows exponentially (as for $f(x)=e^{a x}$ with $\left.a>1\right)$, then $\sum_{r=v}^{\infty}\left(\left|f^{(r)}(0)\right| /(r-v+1)\right.$ !) will be finite for any $v \in \mathbb{N}$. The theorem implies that the Walsh coefficients decay with order $\mathcal{O}\left(b^{-\mu_{v}(k)}\right)$.

- Using Sterling's formula we obtain that

$$
\frac{r !}{(r-v+1) !} \approx(r-v+1)^{v-1}
$$

as $r$ tends to infinity. For $f(x)=1 /(1-c x)$ with $0<c<1$ we have $f_{r}=c^{r}$. In this case we have

$$
\sum_{r=v}^{\infty} \frac{r !\left|f_{r}\right|}{(r-v+1) !} \approx \sum_{r=v}^{\infty}(r-v+1)^{v-1} c^{r}=c^{v-1} \sum_{r=1}^{\infty} r^{v-1} c^{r}<\infty,
$$

for all $v \in \mathbb{N}$. The theorem implies that the Walsh coefficients decay with order $\mathcal{O}\left(b^{-\mu_{v}(k)}\right)$.

For $f \in C^{\infty}([0,1])$ with $f(x)=\sum_{r=0}^{\infty} f_{r} x^{r}$ we define the semi-norm

$$
\|f\|=\sum_{r=1}^{\infty}\left|f_{r}\right|=\sum_{r=1}^{\infty} \frac{\left|f^{(r)}(0)\right|}{r !}
$$

Then the $(v-1)$ th derivative of $f$ is given by

$$
f^{(v-1)}(x)=\sum_{r=0}^{\infty} \frac{(v-1+r) !}{r !} f_{v-1+r} x^{r}=\sum_{r=v-1}^{\infty} \frac{r !}{(r-v+1) !} f_{r} x^{r-v+1}
$$

and

$$
\left\|f^{(v-1)}\right\|=\sum_{r=v}^{\infty} \frac{r !\left|f_{r}\right|}{(r-v+1) !}=\sum_{r=v}^{\infty} \frac{\left|f^{(r)}(0)\right|}{(r-v+1) !} .
$$

Hence, we obtain the following corollary from Theorem 4 .

COROllary 6. Let $f \in C^{\infty}([0,1])$ with $\left\|f^{(z)}\right\|<\infty$ for all $z \in \mathbb{N}_{0}$. Then for every $k \in \mathbb{N}$ we have

$$
|\hat{f}(k)| \leq b^{-\mu_{v}(k)} \frac{3}{(2 \sin (\pi / b))^{v}}\left(1+\frac{1}{b}+\frac{1}{b(b+1)}\right)^{v-1}\left\|f^{(v-1)}\right\| .
$$

Let us consider another example: let $f_{r}=r^{-\delta}$, with $\delta>1$. So, for example, we can choose $u=\min (v,\lceil\delta\rceil-2)$ in the theorem above, which will guarantee that $\sum_{r=v}^{\infty}\left(r !\left|f_{r}\right| /(r-u+1) !\right)<\infty$. On the other hand, this sum is not finite for $\lceil\delta\rceil-2<u \leq v$. The theorem implies that the Walsh coefficients decay with order $\mathcal{O}\left(b^{-\mu_{\min (v,\lceil\delta\rceil-2)}(k)}\right)$. Note that this function $f$ is only $\lceil\delta\rceil-2$ times continuously differentiable. We consider this case in the next section. 


\section{On the Walsh coefficients of functions in $C^{r}([0,1])$}

In this section we prove an explicit constant $C_{r}$, which is better than the constant which can be obtained from [4].

Before the next lemma we introduce a variation of fractional order: For $0<\lambda \leq 1$ and $f:[0,1] \rightarrow \mathbb{R}$ let

$$
V_{\lambda}(f)=\sup _{0=x_{0}<x_{1}<\cdots<x_{N-1}<x_{N}=1} \sum_{n=1}^{N}\left|x_{n}-x_{n-1}\right| \frac{\left|f\left(x_{n}\right)-f\left(x_{n-1}\right)\right|}{\left|x_{n}-x_{n-1}\right|^{\lambda}},
$$

where the supremum is taken over all partitions of the interval $[0,1]$.

If $f$ has a continuous first derivative on $[0,1]$, then

$$
V_{1}(f)=\int_{0}^{1}\left|f^{\prime}(x)\right| d x .
$$

If $f$ satisfies a Hölder condition of order $0<\lambda \leq 1$, that is, $|f(x)-f(y)| \leq$ $C_{f}|x-y|^{\lambda}$ for all $x, y \in[0,1]$, then $V_{\lambda}(f) \leq C_{f}$.

The following lemma appeared already in [6] (albeit in a slightly different form, see also $[4,8])$.

Lemma 7. Let $0<\lambda \leq 1$ and let $f \in \mathcal{L}_{2}([0,1])$ satisfy $V_{\lambda}(f)<\infty$. Then for any $k \in \mathbb{N}$, the $k$ th Walsh coefficient satisfies

$$
|\hat{f}(k)| \leq(b-1)^{1+\lambda} b^{-\lambda a_{1}} V_{\lambda}(f) .
$$

Thus, the decay of the Walsh coefficients of functions with smoothness $0<r \leq 1$ has already been considered and we deal with $r>1$ in the following.

Now let $f \in \mathcal{L}_{2}([0,1])$ with $V_{\lambda}(f)<\infty$ and let $F_{1}(x)=\int_{0}^{x} f(y) d y$. Then using integration by parts as in the previous section, we obtain for $k \in \mathbb{N}$

$$
\hat{F}_{1}(k)=\int_{0}^{1} F_{1}(x) \overline{\operatorname{wal}_{k}(x)} d x=-\int_{0}^{1} f(x) J_{k}(x) d x .
$$

Substituting the Walsh series for $J_{k}$ from Lemma 1, we obtain

$$
\begin{gathered}
\hat{F}_{1}(k)=-b^{-a_{1}}\left(\left(1-\omega_{b}^{-\kappa_{1}}\right)^{-1} \hat{f}\left(k^{\prime}\right)+\left(1 / 2+\left(\omega_{b}^{-\kappa_{1}}-1\right)^{-1}\right) \hat{f}(k)\right. \\
\left.+\sum_{c=1}^{\infty} \sum_{\vartheta=1}^{\infty} b^{-c}\left(\omega_{b}^{\vartheta}-1\right)^{-1} \hat{f}\left(\vartheta b^{a_{1}+c-1}+k\right)\right) .
\end{gathered}
$$

Taking the absolute value on both sides and using the same estimations as in the previous section, we obtain

$$
\left|\hat{F}_{1}(k)\right| \leq \frac{b^{-a_{1}}}{2 \sin (\pi / b)}\left(\left|\hat{f}\left(k^{\prime}\right)\right|+|\hat{f}(k)|+(b-1) \sum_{c=1}^{\infty} b^{-c}\left|\hat{f}\left(\vartheta b^{a_{1}+c-1}+k\right)\right|\right) .
$$


Thus, using Lemma 7 we obtain for $k \in \mathbb{N}$ with $v \geq 2$ that

$$
\left|\hat{F}_{1}(k)\right| \leq b^{-a_{1}-\lambda a_{2}} V_{\lambda}(f) \frac{(b-1)^{1+\lambda}}{2 \sin (\pi / b)}\left(1+2 b^{-\lambda}\right) .
$$

For $k=\kappa_{1} b^{a_{1}-1}$, we obtain

$$
\left|\hat{F}_{1}(k)\right| \leq \frac{b^{-a_{1}}}{2 \sin (\pi / b)}\left(|\hat{f}(0)|+2(b-1)^{1+\lambda} b^{-\lambda a_{1}} V_{\lambda}(f)\right) .
$$

Defining $F_{r}(x)=\int_{0}^{x} F_{r-1}(y) d y$ for $r \geq 1$, we can obtain bounds on the Walsh coefficients of $F_{r}$ by using induction on $r$. Using similar arguments as in the proof of Lemma 3 we obtain for $v>r$ that

$$
\left|\hat{F}_{r}(k)\right| \leq b^{-\mu_{r}(k)-\lambda a_{r+1}} V_{\lambda}(f) \frac{(b-1)^{1+\lambda}\left(1+2 b^{-\lambda}\right)}{(2 \sin (\pi / b))^{r}}\left(1+\frac{1}{b}+\frac{1}{b(b+1)}\right)^{r-1},
$$

and for $v=r$ that

$$
\begin{aligned}
\left|\hat{F}_{r}(k)\right| \leq \frac{b^{-\mu_{r}(k)}}{(2 \sin (\pi / b))^{r}}\left(1+\frac{1}{b}+\frac{1}{b(b+1)}\right)^{r-1} \\
\quad \times\left(|\hat{f}(0)|+2(b-1)^{1+\lambda} b^{-\lambda a_{r}} V_{\lambda}(f)\right) .
\end{aligned}
$$

For $1 \leq v<r$, we have

$$
\begin{aligned}
\left|\hat{F}_{r}(k)\right| \leq \frac{b^{-\mu_{r}(k)}}{(2 \sin (\pi / b))^{v}}\left(1+\frac{1}{b}+\frac{1}{b(b+1)}\right)^{v-1} & \\
& \quad \times\left(\left|\hat{F}_{r-v}(0)\right|+2(b-1)^{1+\lambda} b^{-\lambda a_{v}} V_{\lambda}\left(F_{r-v}\right)\right) .
\end{aligned}
$$

Note that we also have

$$
F_{r}(x)=\int_{0}^{1} f(t) \frac{(x-t)_{+}^{r-1}}{(r-1) !} d t
$$

where

$$
(x-t)_{+}^{r-1}=(x-t)^{r-1} 1_{[0, x)}(t) \text { for } 0 \leq x, t \leq 1 \quad \text { and } \quad 1_{[0, x)}(t) \text { is } 1 \text { for } t \in[0, x)
$$

and zero otherwise.

A function $f \in C^{r}([0,1])$ for which $V_{\lambda}\left(f^{(r)}\right)<\infty$ can be represented by a Taylor series

$$
f(x)=f(0)+\frac{f^{(1)}(0)}{1 !} x+\cdots+\frac{f^{(r-1)}(0)}{(r-1) !} x^{r-1}+\int_{0}^{1} f^{(r)}(t) \frac{(x-t)_{+}^{r-1}}{(r-1) !} d t .
$$


With this we can now obtain a bound on the Walsh coefficients of $f$. For $v \geq r$ we know from [4] that

$$
\int_{0}^{1}\left(f(0)+\frac{f^{(1)}(0)}{1 !} x+\cdots+\frac{f^{(r-1)}(0)}{(r-1) !} x^{r-1}\right) \overline{\operatorname{wal}_{k}(x)} d x=0 .
$$

To bound the Walsh coefficient of

$$
\int_{0}^{1} f^{(r)}(t) \frac{(x-t)_{+}^{r-1}}{(r-1) !} d t
$$

for $v>r$ we can use (7) to obtain

$$
|\hat{f}(k)| \leq b^{-\mu_{r}(k)-\lambda a_{r+1}} V_{\lambda}\left(f^{(r)}\right) \frac{(b-1)^{1+\lambda}\left(1+2 b^{-\lambda}\right)}{(2 \sin (\pi / b))^{r}}\left(1+\frac{1}{b}+\frac{1}{b(b+1)}\right)^{r-1} .
$$

For $v=r$ we can use (8) to obtain

$$
\begin{aligned}
|\hat{f}(k)| \leq \frac{b^{-\mu_{r}(k)}}{(2 \sin (\pi / b))^{r}}\left(1+\frac{1}{b}+\frac{1}{b(b+1)}\right)^{r-1} \\
\quad \times\left(\left|\hat{f}^{(r)}(0)\right|+2(b-1)^{1+\lambda} b^{-\lambda a_{r}} V_{\lambda}\left(f^{(r)}\right)\right) .
\end{aligned}
$$

For $1 \leq v<r$ we have

$$
\begin{aligned}
& \left|\int_{0}^{1}\left(f(0)+\frac{f^{(1)}(0)}{1 !} x+\cdots+\frac{f^{(r-1)}(0)}{(r-1) !} x^{r-1}\right) \overline{\operatorname{wal}_{k}(x)} d x\right| \\
& \quad \leq b^{-\mu_{r}(k)} \frac{3}{(2 \sin (\pi / b))^{v}}\left(1+\frac{1}{b}+\frac{1}{b(b+1)}\right)^{v-1} \sum_{s=v}^{r-1} \frac{\left|f^{(s)}(0)\right|}{(s-v+1) !}
\end{aligned}
$$

and, therefore, using (9), we obtain

$$
\begin{aligned}
& |\hat{f}(k)| \leq \frac{b^{-\mu_{r}(k)}}{(2 \sin (\pi / b))^{v}}\left(1+\frac{1}{b}+\frac{1}{b(b+1)}\right)^{v-1} \\
& \quad \times\left[3 \sum_{s=v}^{r-1} \frac{\left|f^{(s)}(0)\right|}{(s-v+1) !}+\left(\left|\hat{f}^{(v)}(0)\right|+2(b-1)^{1+\lambda} b^{-\lambda a_{v}} V_{\lambda}\left(f^{(v)}\right)\right)\right],
\end{aligned}
$$

where $\hat{f}^{(v)}(0)$ denotes the zeroth Walsh coefficient of $f^{(v)}$. We have shown the following theorem.

THEOREM 8. Let $f \in C^{r}([0,1])$ with $V_{\lambda}\left(f^{(r)}\right)<\infty$, and let $k \in \mathbb{N}$. Then, for $v>r$, we have

$$
|\hat{f}(k)| \leq b^{-\mu_{r}(k)-\lambda a_{r+1}} V_{\lambda}\left(f^{(r)}\right) \frac{(b-1)^{1+\lambda}\left(1+2 b^{-\lambda}\right)}{(2 \sin (\pi / b))^{r}}\left(1+\frac{1}{b}+\frac{1}{b(b+1)}\right)^{r-1},
$$


for $r=v$

$$
\begin{aligned}
& |\hat{f}(k)| \leq \frac{b^{-\mu_{r}(k)}}{(2 \sin (\pi / b))^{r}}\left(1+\frac{1}{b}+\frac{1}{b(b+1)}\right)^{r-1} \\
& \quad \times\left(\left|\int_{0}^{1} f^{(r)}(x) d x\right|+2(b-1)^{1+\lambda} b^{-\lambda a_{r}} V_{\lambda}\left(f^{(r)}\right)\right),
\end{aligned}
$$

and, for $v<r$, we have

$$
\begin{array}{r}
|\hat{f}(k)| \leq \frac{b^{-\mu_{r}(k)}}{(2 \sin (\pi / b))^{v}}\left(1+\frac{1}{b}+\frac{1}{b(b+1)}\right)^{v-1}\left[3 \sum_{s=v}^{r-1} \frac{\left|f^{(s)}(0)\right|}{(s-v+1) !}\right. \\
\left.+\left(\left|\int_{0}^{1} f^{(v)}(x) d x\right|+2(b-1)^{1+\lambda} b^{-\lambda a_{v}} V_{\lambda}\left(f^{(v)}\right)\right)\right] .
\end{array}
$$

We also prove bounds on the decay of the Walsh coefficients of functions from Sobolev spaces. For this, we first need bounds on the Walsh coefficients of Bernoulli polynomials, which we consider in the next section.

\section{On the Walsh coefficients of Bernoulli polynomials}

For $r \geq 0$, let $B_{r}(\cdot)$ denote the Bernoulli polynomial of degree $r$ and $b_{r}(\cdot)=$ $B_{r}(\cdot) / r$ !. For example, we have $B_{0}(x)=1, B_{1}(x)=x-1 / 2, B_{2}(x)=x^{2}-x+1 / 6$ and so on. Those polynomials have the properties

$$
b_{r}^{\prime}(x)=b_{r-1}(x) \quad \text { and } \quad \int_{0}^{1} b_{r}(x)=0 \quad \text { for all } r \geq 1 .
$$

We obviously have $b_{0}^{\prime}(x)=0$ and $\int_{0}^{1} b_{0}(x) d x=1$. Further, $B_{r}(1-x)=(-1)^{r} B_{r}(x)$ and also $b_{r}(1-x)=(-1)^{r} b_{r}(x)$. The numbers $B_{r}=B_{r}(0)$ are the Bernoulli numbers and $B_{r}=0$ for all odd $r \geq 3$. Further, for $r \geq 2$,

$$
b_{r}(x)=-\frac{1}{(2 \pi i)^{r}} \sum_{h \in \mathbb{Z} \backslash\{0\}} h^{-r} e^{2 \pi i h x} \quad \text { for } 0 \leq x \leq 1 .
$$

It is more convenient to calculate with $b_{r}(\cdot)$ rather than the Bernoulli polynomials.

For $r \geq 1$ and $k \in \mathbb{N}$, let

$$
\beta_{r, v}\left(a_{1}, \ldots, a_{v} ; \kappa_{1}, \ldots, \kappa_{v}\right)=\int_{0}^{1} b_{r}(x) \overline{\operatorname{wal}_{k}(x)} d x
$$

As for $\chi_{r, v}$, we also have $\beta_{r, v}=0$ for $v>r$. Further, for $k=0$ let $v=0$ and we have $\beta_{r, 0}=0$ for all $r \geq 1$. 
The Walsh series for $b_{1}$ can be obtained from the Walsh series of $J_{0}$ from Lemma 1 and is given by

$$
b_{1}(x)=x-1 / 2=\sum_{c=1}^{\infty} \sum_{\vartheta=1}^{b-1} b^{-c}\left(\omega_{b}^{-\vartheta}-1\right)^{-1} \mathrm{wal}_{\vartheta b^{c-1}}(x) .
$$

Thus,

$$
\beta_{1,1}\left(a_{1} ; \kappa_{1}\right)=-b^{-a_{1}}\left(1-\omega_{b}^{-\kappa_{1}}\right)^{-1} .
$$

Using integration by parts and $J_{k}(0)=J_{k}(1)=0$ we obtain for all $r>1$ that

$$
\int_{0}^{1} b_{r}(x) \overline{\mathrm{wal}_{k}(x)} d x=-\int_{0}^{1} b_{r-1}(x) J_{k}(x) d x .
$$

Using Lemma 1 and (11) we obtain for $1 \leq v \leq r$ and $r>1$ that

$$
\begin{aligned}
\beta_{r, v}\left(a_{1}, \ldots, a_{v} ; \kappa_{1}, \ldots, \kappa_{v}\right) & \\
=- & b^{-a_{1}}\left(\left(1-\omega_{b}^{-\kappa_{1}}\right)^{-1} \beta_{r-1, v-1}\left(a_{2}, \ldots, a_{v} ; \kappa_{2}, \ldots, \kappa_{v}\right)\right. \\
& +\left(1 / 2+\left(\omega_{b}^{-\kappa_{1}}-1\right)^{-1}\right) \beta_{r-1, v}\left(a_{1}, \ldots, a_{v} ; \kappa_{1}, \ldots, \kappa_{v}\right) \\
& +\sum_{c=1}^{\infty} \sum_{\vartheta=1}^{b-1} b^{-c}\left(\omega_{b}^{\vartheta}-1\right)^{-1} \beta_{r-1, v+1} \\
& \left.\times\left(a_{1}+c, a_{1}, \ldots, a_{v} ; \vartheta, \kappa_{1}, \ldots, \kappa_{v}\right)\right) .
\end{aligned}
$$

From (12) we can obtain

$$
\beta_{r, r}\left(a_{1}, \ldots, a_{r} ; \kappa_{1}, \ldots, \kappa_{r}\right)=(-1)^{r} b^{-a_{1}-\cdots-a_{r}} \prod_{s=1}^{r}\left(1-\omega_{b}^{-\kappa_{s}}\right)^{-1}
$$

for all $r \geq 1$.

The first few values of $\beta_{r, v}$ are as follows:

- $\quad r=1: \beta_{1,0}=0, \beta_{1,1}\left(a_{1} ; \kappa_{1}\right)=-b^{-a_{1}}\left(1-\omega_{b}^{-\kappa_{1}}\right)^{-1}$;

- $r=2: \beta_{2,0}=0, \beta_{2,1}\left(a_{1} ; \kappa_{1}\right)=b^{-2 a_{1}}\left(1-\omega_{b}^{-\kappa_{1}}\right)^{-1}\left(1 / 2+\left(\omega_{b}^{-\kappa_{1}}-1\right)^{-1}\right)$,

$\beta_{2,2}\left(a_{1}, a_{2} ; \kappa_{1}, \kappa_{2}\right)=b^{-a_{1}-a_{2}}\left(1-\omega_{b}^{-\kappa_{1}}\right)^{-1}\left(1-\omega_{b}^{-\kappa_{2}}\right)^{-1}$.

In principle we can obtain all values of $\beta_{r, v}$ recursively using (12). We have already calculated $\beta_{r, v}$ for $v=r$ and we could continue doing so for $v=r-1, \ldots, 1$. However, the formulae become increasingly complex, so we only prove a bound on them.

For any $r \geq 0$ and a nonnegative integer $k$, we introduce the function

$$
\mu_{r, \operatorname{per}}(k)= \begin{cases}0 & \text { for } r=0, k \geq 0, \\ 0 & \text { for } k=0, r \geq 0, \\ a_{1}+\cdots+a_{v}+(r-v) a_{v} & \text { for } 1 \leq v<r \\ a_{1}+\cdots+a_{r} & \text { for } v \geq r\end{cases}
$$


LEMMA 9. For any $r \geq 2$ and $1 \leq v \leq r$ we have

$$
\left|\beta_{r, v}\left(a_{1}, \ldots, a_{v} ; \kappa_{1}, \ldots, \kappa_{v}\right)\right| \leq \frac{b^{-\mu_{r, \mathrm{per}}(k)}}{(2 \sin (\pi / b))^{r}}\left(1+\frac{1}{b}+\frac{1}{b(b+1)}\right)^{r-2} .
$$

PROOF. We prove the bound by induction on $r$. Using Lemma 2 it can easily be seen that the result holds for $r=2$. Hence, assume now that $r>2$ and the result holds for $r-1$. By taking the absolute value of (12) and using the triangular inequality together with Lemma 2 we obtain

$$
\begin{aligned}
& \left|\beta_{r, v}\left(a_{1}, \ldots, a_{v} ; \kappa_{1}, \ldots, \kappa_{v}\right)\right| \\
& \leq \frac{b^{-a_{1}}}{2 \sin (\pi / b)}\left(\left|\beta_{r-1, v-1}\left(a_{2}, \ldots, a_{v} ; \kappa_{2}, \ldots, \kappa_{v}\right)\right|\right. \\
& \quad+\left|\beta_{r-1, v}\left(a_{1}, \ldots, a_{v} ; \kappa_{1}, \ldots, \kappa_{v}\right)\right| \\
& \left.\quad+\sum_{c=1}^{\infty} \sum_{\vartheta=1}^{b-1} b^{-c}\left|\beta_{r-1, v+1}\left(a_{1}+c, a_{1}, \ldots, a_{v} ; \vartheta, \kappa_{1}, \ldots, \kappa_{v}\right)\right|\right) .
\end{aligned}
$$

We can now use the induction assumption for $\left|\beta_{r-1, v-1}\right|,\left|\beta_{r-1, v}\right|,\left|\beta_{r-1, v+1}\right|$. Hence, for $v>1$, we obtain

$$
\begin{aligned}
& \left|\beta_{r, v}\left(a_{1}, \ldots, a_{v} ; \kappa_{1}, \ldots, \kappa_{v}\right)\right| \\
& \quad \leq \frac{b^{-\mu_{r, \mathrm{per}}(k)}}{(2 \sin (\pi / b))^{r}}\left(1+\frac{1}{b}+\frac{1}{b(b+1)}\right)^{r-3}\left(1+b^{a_{2}-a_{1}}+\sum_{c=1}^{\infty} \sum_{\vartheta=1}^{b-1} b^{-2 c} b^{a_{2}-a_{1}}\right) .
\end{aligned}
$$

By noting that $\sum_{c=1}^{\infty} \sum_{\vartheta=1}^{b-1} b^{-2 c}=(1 /(b+1))$, and $a_{1}>a_{2}$ we obtain the result.

For $v=1$ note that $\beta_{r, 0}=0$. In this case we have

$$
\begin{aligned}
\left|\beta_{r, 1}\left(a_{1} ; \kappa_{1}\right)\right| & \leq \frac{b^{-\mu_{r, \text { per }}(k)}}{(2 \sin (\pi / b))^{r}}\left(1+\frac{1}{b}+\frac{1}{b(b+1)}\right)^{r-3}\left(1+\sum_{c=1}^{\infty} \sum_{\vartheta=1}^{b-1} b^{-2 c}\right) \\
& \leq \frac{b^{-\mu_{r, \text { per }}(k)}}{(2 \sin (\pi / b))^{r}}\left(1+\frac{1}{b}+\frac{1}{b(b+1)}\right)^{r-2},
\end{aligned}
$$

which implies the result.

The $b_{r}$ are polynomials, but using (10) we can extend $b_{r}$ periodically so that it is defined on $\mathbb{R}$. We denote those functions by $\widetilde{b}_{r}$. Then, for $r \geq 1$,

$$
\tilde{b}_{2 r}(x)=\frac{2(-1)^{r+1}}{(2 \pi)^{2 r}} \sum_{h=1}^{\infty} h^{-2 r} \cos 2 \pi h x \quad \text { for } x \in \mathbb{R},
$$

and

$$
\tilde{b}_{2 r+1}(x)=\frac{2(-1)^{r+1}}{(2 \pi)^{2 r+1}} \sum_{h=1}^{\infty} h^{-2 r-1} \sin 2 \pi h x \quad \text { for } x \in \mathbb{R} .
$$


From this it can be seen that $\widetilde{b}_{r}(x)=(-1)^{r} \widetilde{b}_{r}(-x)$ for all $r \geq 2$. Note that for $x, y \in[0,1] \quad$ we have $b_{2 r}(|x-y|)=\tilde{b}_{2 r}(x-y) \quad$ and $\quad b_{2 r+1}(|x-y|)=$ $(-1)^{1_{x<y}} \widetilde{b}_{2 r+1}(x-y)$, where $1_{x<y}$ is one for $x<y$ and zero otherwise. We also extend $B_{r}(\cdot)$ periodically to $\mathbb{R}$, which we denote by $\widetilde{B}_{r}(\cdot)$.

In the next section we also need a bound on the Walsh coefficients of $\widetilde{b}_{r}(x-y)$. For $k, l \geq 0$, let

$$
\begin{aligned}
\gamma_{r}(k, l) & =\int_{0}^{1} \int_{0}^{1} \tilde{b}_{r}(x-y) \overline{\operatorname{wal}_{k}(x)} \operatorname{wal}_{l}(y) d x d y \\
& =-\frac{1}{(2 \pi i)^{r}} \sum_{h \in \mathbb{Z} \backslash\{0\}} h^{-r} \tau_{h, k} \overline{\tau_{h, l}},
\end{aligned}
$$

where

$$
\tau_{h, k}=\int_{0}^{1} e^{2 \pi i h x} \overline{\operatorname{wal}_{k}(x)} d x .
$$

We have $\gamma_{r}(k, 0)=\gamma_{r}(0, l)=0$ for all $k, l \geq 0$, as $\int_{z}^{1+z} \widetilde{b}_{r}(x) d x=0$ for any $z \in \mathbb{R}$. Further we have $\gamma_{r}(l, k)=(-1)^{r} \overline{\gamma_{r}(k, l)}$ and therefore also $\left|\gamma_{r}(k, l)\right|=\left|\gamma_{r}(l, k)\right|$.

We obtain bounds on $\gamma_{r}$ by induction. In the next lemma we calculate the values of $\gamma_{2}$.

LEMMA 10. For all $k, l \geq 0$ we have $\gamma_{2}(k, 0)=\gamma_{2}(0, l)=0$. For $k, l>0$ we have

$$
\gamma_{2}(k, l)= \begin{cases}b^{-2 a_{1}}\left(\frac{1}{2 \sin ^{2} \kappa_{1} \pi / b}-\frac{1}{3}\right) & \text { if } k=l, \\ b^{-a_{1}-d_{1}}\left(\omega_{b}^{-\kappa_{1}}-1\right)^{-1}\left(\omega_{b}^{\lambda_{1}}-1\right)^{-1} & \text { if } k^{\prime}=l^{\prime}>0, \\ b^{-a_{1}-d_{1}}\left(1 / 2+\left(\omega_{b}^{-\lambda_{1}}-1\right)^{-1}\right)\left(\omega_{b}^{-\kappa_{1}}-1\right)^{-1} & \text { and } k \neq l, \\ +b^{-2 a_{1}}\left(1 / 2+\left(\omega_{b}^{\kappa_{1}}-1\right)^{-1}\right)\left(1-\omega_{b}^{-\kappa_{1}}\right)^{-1} & \text { if } k^{\prime}=l, \\ b^{-a_{1}-d_{1}}\left(1 / 2+\left(\omega_{b}^{\kappa_{1}}-1\right)^{-1}\right)\left(\omega_{b}^{\lambda_{1}}-1\right)^{-1} & \\ +b^{-2 d_{1}}\left(1 / 2+\left(\omega_{b}^{-\lambda_{1}}-1\right)^{-1}\right)\left(1-\omega_{b}^{\lambda_{1}}\right)^{-1} & \text { if } k=l^{\prime}, \\ b^{-a_{1}-a_{2}}\left(1-\omega_{b}^{-\kappa_{2}}\right)^{-1}\left(\omega_{b}^{-\kappa_{1}}-1\right)^{-1} & \text { if } k^{\prime \prime}=l, \\ b^{-d_{1}-d_{2}}\left(1-\omega_{b}^{\lambda_{2}}\right)^{-1}\left(\omega_{b}^{\lambda_{1}}-1\right)^{-1} & \text { if } k=l^{\prime \prime}, \\ 0 & \text { otherwise. }\end{cases}
$$

PROOF. Note that $\gamma_{2}(k, 0)=\gamma_{2}(0, l)=0$ for all $k, l \geq 0$, as $\int_{z}^{1+z} \tilde{b}_{2}(x) d x=0$ for any $z \in \mathbb{R}$.

Now assume that $k, l>0$. The value of $\gamma_{2}(k, k)$ has been obtained in [5, Appendix A] (but can also be obtained from the following).

The Walsh series for

$$
\tilde{b}_{2}(x-y)=b_{2}(|x-y|)=\frac{(x-y)^{2}}{2}-\frac{|x-y|}{2}+\frac{1}{6}
$$


can be calculated in the following way: we have $x=\overline{J_{0}(x)}$ and $y=J_{0}(y)$ and so

Furthermore,

$$
\frac{(x-y)^{2}}{2}=\frac{\left(\overline{J_{0}(x)}-J_{0}(y)\right)^{2}}{2} .
$$

$$
|x-y|=x+y-2 \min (x, y)=x+y-2 \int_{0}^{1} 1_{[0, x)}(t) 1_{[0, y)}(t) d t,
$$

where $1_{[0, x)}(t)$ is one for $t \in[0, x)$ and zero otherwise. Note that $J_{k}(x)=$ $\int_{0}^{x} \overline{\operatorname{wal}_{k}(t)} d t=\int_{0}^{1} 1_{[0, x)}(t) \overline{\operatorname{wal}_{k}(t)} d t$, which implies

$$
1_{[0, x)}(t)=\sum_{k=0}^{\infty} J_{k}(x) \mathrm{wal}_{k}(t)
$$

Thus,

$$
\begin{aligned}
\min (x, y) & =\int_{0}^{1} 1_{[0, x)}(t) 1_{[0, y)}(t) d t \\
& =\sum_{m, n=0}^{\infty} \overline{J_{m}(x)} J_{n}(y) \int_{0}^{1} \overline{\operatorname{wal}_{m}(t)} \mathrm{wal}_{n}(t) d t \\
& =\sum_{m=0}^{\infty} \overline{J_{m}(x)} J_{m}(y) .
\end{aligned}
$$

The Walsh series for $\tilde{b}_{2}(x-y)$ is therefore given by

$$
\tilde{b}_{2}(x-y)=\frac{\left(\overline{J_{0}(x)}\right)^{2}+\left(J_{0}(y)\right)^{2}-\overline{J_{0}(x)}-J_{0}(y)}{2}+\sum_{m=1}^{\infty} \overline{J_{m}(x)} J_{m}(y)+\frac{1}{6} \text {. }
$$

We have

$$
\begin{aligned}
\gamma_{2}(k, l) & =\int_{0}^{1} \int_{0}^{1} \tilde{b}_{2}(x-y) \overline{\operatorname{wal}_{k}(x)} \mathrm{wal}_{l}(y) d x d y \\
& =\int_{0}^{1} \int_{0}^{1}\left[\frac{\left(\overline{J_{0}(x)}\right)^{2}+\left(J_{0}(y)\right)^{2}-\overline{J_{0}(x)}-J_{0}(y)}{2}+\sum_{m=1}^{\infty} \overline{J_{m}(x)} J_{m}(y)+\frac{1}{6}\right] \\
& \times \overline{\operatorname{wal}_{k}(x)} \operatorname{wal}_{l}(y) d x d y \\
& =\sum_{m=1}^{\infty} \int_{0}^{1} \overline{J_{m}(x) \operatorname{wal}_{k}(x)} d x \int_{0}^{1} J_{m}(y) \operatorname{wal}_{l}(y) d y
\end{aligned}
$$

It remains to consider the integral $\int_{0}^{1} \overline{J_{m}(x) \mathrm{wal}_{k}(x)} d x$. Let $m=\eta b^{e-1}+m^{\prime}$, with $0<\eta<b, e>0$, and $0 \leq m^{\prime}<b^{e-1}$. Then

$$
\begin{aligned}
& \int_{0}^{1} \overline{J_{m}(x) \mathrm{wal}_{k}(x)} d x \\
& \quad=b^{-e}\left(\left(1-\omega_{b}^{\eta}\right)^{-1} \int_{0}^{1} \operatorname{wal}_{m^{\prime}}(x) \overline{\operatorname{wal}_{k}(x)} d x\right.
\end{aligned}
$$




$$
\begin{aligned}
& +\left(1 / 2+\left(\omega_{b}^{\eta}-1\right)^{-1}\right) \int_{0}^{1} \operatorname{wal}_{m}(x) \overline{\mathrm{wal}_{k}(x)} d x \\
& \left.+\sum_{c=1}^{\infty} \sum_{\vartheta=1}^{b-1} b^{-c}\left(\omega_{b}^{-\vartheta}-1\right)^{-1} \int_{0}^{1} \operatorname{wal}_{\vartheta b^{e+c-1}+m}(x) \overline{\operatorname{wal}_{k}(x)} d x\right) .
\end{aligned}
$$

This integral is not zero if and only if either $m^{\prime}=k, m=k$ or $m+\vartheta b^{e+c-1}=k$ for some $\vartheta, c$. Analogously the same applies to the integral $\int_{0}^{1} J_{m}(y) \mathrm{wal}_{l}(y) d y$. Hence, we only need to consider a few cases for which $\gamma_{2}(k, l)$ is nonzero, and by going through each of them we obtain the result.

Note that many values for $\gamma_{2}(k, l)$ are zero, in particular, if $k$ and $l$ are sufficiently 'different' from each other. This property is inherited by $b_{r}$ for $r>2$ via the recursion

$$
\begin{aligned}
\gamma_{r}(k, l)=-b^{-a_{1}} & \left(\left(1-\omega_{b}^{-\kappa_{1}}\right)^{-1} \gamma_{r-1}\left(k^{\prime}, l\right)+\left(1 / 2+\left(\omega_{b}^{-\kappa}-1\right)^{-1}\right) \gamma_{r-1}(k, l)\right. \\
& \left.+\sum_{c=1}^{\infty} \sum_{\vartheta=1}^{b-1} b^{-c}\left(\omega_{b}^{\vartheta}-1\right)^{-1} \gamma_{r-1}\left(\vartheta b^{c+a_{1}-1}+k, l\right)\right) .
\end{aligned}
$$

This recursion is obtained from

$$
\gamma_{r}(k, l)=-\int_{0}^{1} \int_{0}^{1} \tilde{b}_{r-1}(x-y) J_{k}(x) \operatorname{wal}_{l}(y) d x d y,
$$

which in turn can be obtained using integration by parts. In the following lemma we show that $\gamma_{r}(k, l)=0$ for many choices of $k$ and $l$.

LEMMA 11. We have the following results.

(1) For any $k, l \geq 0$ we have $\gamma_{r}(k, 0)=\gamma_{r}(0, l)=0$.

(2) For $k, l>0$ with $|v-w|>r$ we have $\gamma_{r}(k, l)=0$.

(3) Let $k, l>0$ such that $|v-w| \leq r$.

(i) If $v=1$, but $\left(\kappa_{1}, a_{1}\right) \neq\left(\lambda_{w}, d_{w}\right)$, then $\gamma_{r}(k, l)=0$.

(ii) If $w=1$, but $\left(\lambda_{1}, d_{1}\right) \neq\left(\kappa_{v}, a_{v}\right)$, then $\gamma_{r}(k, l)=0$.

(iii) If $r-1 \leq|v-w| \leq r$, but

$$
\begin{aligned}
& \left(a_{v-\min }(v, w)+1, \ldots, a_{v}, \kappa_{v-\min }(v, w)+1, \ldots, \kappa_{v}\right) \\
& \neq\left(d_{w-\min (v, w)+1}, \ldots, d_{w}, \lambda_{w-\min (v, w)+1}, \ldots, \lambda_{w}\right),
\end{aligned}
$$

then $\gamma_{r}(k, l)=0$.

(iv) If $v, w>1$ and $0 \leq|v-w| \leq r-2$, but

$$
\begin{aligned}
& \left(a_{v-\min }(v, w)+2, \ldots, a_{v}, \kappa_{v-\min }(v, w)+2, \ldots, \kappa_{v}\right) \\
& \quad \neq\left(d_{w-\min }(v, w)+2, \ldots, d_{w}, \lambda_{w-\min }(v, w)+2, \ldots, \lambda_{w}\right),
\end{aligned}
$$

then $\gamma_{r}(k, l)=0$.

PROOF. (a) This follows from $\int_{z}^{z+1} \widetilde{b}_{r}(x) d x=0$ for all $z \in \mathbb{R}$.

(b) We have $\gamma_{2}(k, l)=0$ for $|v-w|>2$, which follows from Lemma 10. Let $r>2$.

Then by repeatedly using (13) we can write $\gamma_{r}(k, l)$ as a sum of $\gamma_{2}\left(m_{i}, n_{j}\right)$ 
for some values $m_{i}, n_{j}$; that is, $\gamma_{r}(k, l)=\sum_{i, j} a_{i, j} \gamma_{2}\left(m_{i}, n_{j}\right)$. However, if $|v-w|>2$, then the difference between the number of digits of $m_{i}$ and $n_{j}$ will be larger than two and, hence, $\gamma_{r}(k, l)=0$ by Lemma 10 .

(c) For $r=2$ the proof follows again from Lemma 10: if $v=1(w=1)$, then $k^{\prime}=0\left(l^{\prime}=0\right.$ respectively) and we only have the cases $k=l, k=l^{\prime}\left(l=k^{\prime}\right.$, respectively), and $k=l^{\prime \prime}$ ( $l=k^{\prime \prime}$, respectively) for which the result follows. The case $1 \leq|v-w| \leq 2$ comprises the cases $k^{\prime}=l, k=l^{\prime}, k^{\prime \prime}=l$, and $k=l^{\prime \prime}$. The case $v=w$ can be obtained by considering $k=l$, and $k^{\prime}=l^{\prime}$ with $k \neq l$. For $r>2$, we can again use (13) repeatedly to obtain a sum of $\gamma_{2}\left(m_{i}, n_{j}\right)$. The result then follows by using Lemma 10.

In the following we prove a bound on $\left|\gamma_{r}(k, l)\right|$ for arbitrary $r \geq 2$. We set

$$
\mu_{r, \operatorname{per}}(k, l)=\max _{0 \leq s \leq r} \mu_{s, \operatorname{per}}(k)+\mu_{r-s, \operatorname{per}}(l) .
$$

LEMMA 12. For $r \geq 2$ and $k, l>0$ we have

$$
\left|\gamma_{r}(k, l)\right| \leq \frac{2 b^{-\mu_{r, \mathrm{per}}(k, l)}}{(2 \sin (\pi / b))^{r}}\left(1+\frac{1}{b}+\frac{1}{b(b+1)}\right)^{r-2} .
$$

PROOF. For $r=2$ we use Lemma 10 , and $\left|1 / 2+\left(\omega_{b}^{-\kappa}-1\right)^{-1}\right|,\left|\omega_{b}^{-\kappa}-1\right|^{-1} \leq$ $(2 \sin (\pi / b))^{-1}$ to obtain the result.

Let now $r>2$. By taking the absolute value of (13) and using the triangular inequality together with $\left|1 / 2+\left(\omega_{b}^{-\kappa}-1\right)^{-1}\right|,\left|\omega_{b}^{-\kappa}-1\right|^{-1} \leq(2 \sin (\pi / b))^{-1}$ we obtain

$$
\begin{aligned}
& \left|\gamma_{r}(k, l)\right| \leq \frac{b^{-a_{1}}}{2 \sin (\pi / b)}\left(\left|\gamma_{r-1}\left(k^{\prime}, l\right)\right|+\left|\gamma_{r-1}(k, l)\right|\right. \\
& \left.\quad+\sum_{c=1}^{\infty} \sum_{\vartheta=1}^{b-1} b^{-c}\left|\gamma_{r-1}\left(\vartheta b^{a_{1}+c-1}+k, l\right)\right|\right) .
\end{aligned}
$$

By using integration by parts with respect to the variable $y$ in (13) we obtain a similar formula to (13). Hence, there is also an analogue to (14).

Without loss of generality, assume that $k \geq l$ (otherwise use the analogue to (14)) and assume that the result holds for $r-1$. Then

$$
\begin{aligned}
&\left|\gamma_{r}(k, l)\right| \leq \frac{2 b^{-a_{1}}}{(2 \sin (\pi / b))^{r}}\left(1+\frac{1}{b}+\frac{1}{b(b+1)}\right)^{r-3} \\
& \quad \times\left(b^{-\mu_{r-1, \operatorname{per}}\left(k^{\prime}, l\right)}+b^{-\mu_{r-1, \operatorname{per}}(k, l)}\right. \\
&\left.+(b-1) \sum_{c=1}^{\infty} b^{-c-\mu_{r-1, \operatorname{per}}\left(b^{a_{1}+c-1}+k, l\right)}\right) .
\end{aligned}
$$

We have $a_{1}+\mu_{r-1, \operatorname{per}}\left(k^{\prime}, l\right)=\mu_{r, \text { per }}(k, l), a_{1}+\mu_{r-1, \operatorname{per}}(k, l)>\mu_{r, \operatorname{per}}(k, l)$, and

$$
a_{1}+\mu_{r-1, \operatorname{per}}\left(b^{a_{1}+c-1}+k, l\right)=2 a_{1}+c+\mu_{r-2, \operatorname{per}}(k, l)>c+\mu_{r, \operatorname{per}}(k, l) .
$$


Therefore, we obtain

$$
\left|\gamma_{r}(k, l)\right| \leq \frac{2 b^{-\mu_{r, \text { per }}(k, l)}}{(2 \sin (\pi / b))^{r}}\left(1+\frac{1}{b}+\frac{1}{b(b+1)}\right)^{r-3}\left(1+\frac{1}{b}+\frac{b-1}{b} \sum_{c=1}^{\infty} b^{-2 c}\right) .
$$

As $\sum_{c=1}^{\infty} b^{-2 c}=\left(b^{2}-1\right)^{-1}$, the result follows.

\section{On the Walsh coefficients of functions in Sobolev spaces}

In this section we consider functions in reproducing kernel Hilbert spaces. We consider the Sobolev space $\mathcal{H}_{r}$ of real valued functions $f:[0,1] \rightarrow \mathbb{R}$, for which $r>1$, and where the inner product is given by

$$
\langle f, g\rangle_{r}=\sum_{s=0}^{r-1} \int_{0}^{1} f^{(s)}(x) d x \int_{0}^{1} g^{(s)}(x) d x+\int_{0}^{1} f^{(r)}(x) g^{(r)}(x) d x,
$$

where $f^{(s)}$ denotes the $s$ th derivative of $f$ and where $f^{(0)}=f$. Let $\|f\|_{r}=\sqrt{\langle f, f\rangle_{r}}$. The reproducing kernel (see [1] for more information about reproducing kernels) for this space is given by

$$
\begin{aligned}
\mathcal{K}_{r}(x, y) & =\sum_{s=0}^{r} \frac{B_{s}(x) B_{s}(y)}{(s !)^{2}}-(-1)^{r} \frac{\widetilde{B}_{2 r}(x-y)}{(2 r) !} \\
& =\sum_{s=0}^{r} b_{s}(x) b_{s}(y)-(-1)^{r} \tilde{b}_{2 r}(x-y),
\end{aligned}
$$

see, for example, [10, Section 10.2]. It can be checked that

$$
\begin{aligned}
f(y) & =\left\langle f, \mathcal{K}_{r}(\cdot, y)\right\rangle_{r} \\
& =\sum_{s=0}^{r} \int_{0}^{1} f^{(s)}(x) d x b_{s}(y)-(-1)^{r} \int_{0}^{1} f^{(r)}(x) \widetilde{b}_{r}(x-y) d x .
\end{aligned}
$$

A bound on the Walsh coefficients of $b_{0}(y), \ldots, b_{r}(y)$ can be obtained from Lemma 9. For the remaining term we use Lemma 12. We have

$$
\tilde{b}_{r}(x-y)=\sum_{k, l=1}^{\infty} \gamma_{r}(k, l) \mathrm{wal}_{k}(x) \overline{\operatorname{wal}_{l}(y)}
$$

and therefore the $m$ th Walsh coefficient for the last term is given by

$$
\begin{aligned}
(-1)^{r} & \int_{0}^{1} \int_{0}^{1} f^{(r)}(x) \tilde{b}_{r}(x-y) d x \overline{\operatorname{wal}_{m}(y)} d y \\
& =(-1)^{r} \sum_{k, l=1}^{\infty} \overline{\gamma_{r}(k, l)} \int_{0}^{1} f^{(r)}(x) \overline{\operatorname{wal}_{k}(x)} d x \int_{0}^{1} \operatorname{wal}_{l}(y) \overline{\mathrm{wal}_{m}(y)} d y \\
& =\sum_{k=1}^{\infty} \gamma_{r}(m, k) \int_{0}^{1} f^{(r)}(x) \overline{\operatorname{wal}_{k}(x)} d x .
\end{aligned}
$$


We can estimate the absolute value of the last expression by

$$
\begin{aligned}
& \sum_{k=1}^{\infty}\left|\gamma_{r}(m, k)\right| \int_{0}^{1}\left|f^{(r)}(x)\right| d x \\
& \leq \int_{0}^{1}\left|f^{(r)}(x)\right| d x \frac{2}{(2 \sin (\pi / b))^{r}} \\
& \quad \times\left(1+\frac{1}{b}+\frac{1}{b(b+1)}\right)^{r-2} \sum_{\substack{k=1 \\
\gamma_{r}(m, k) \neq 0}}^{\infty} b^{-\mu_{r, p e r}(m, k)} .
\end{aligned}
$$

It remains to prove a bound on the rightmost sum, which we do in the following lemma.

LEMMA 13. For any $r>1$ and $m \in \mathbb{N}$

$$
\sum_{\substack{k=1 \\ \gamma_{r}(m, k) \neq 0}}^{\infty} b^{-\mu_{r, \mathrm{per}}(m, k)} \leq b^{-\mu_{r, \mathrm{per}}(m)}\left(3+\frac{2}{b}+\frac{2 b+1}{b-1}\right) .
$$

Proof. Let $m=\eta_{1} b^{e_{1}-1}+\cdots+\eta_{z} b^{e_{z}-1}$, where $0<\eta_{1}, \ldots, \eta_{z}<b$ and $e_{1}>$ $\cdots>e_{z}>0$. We consider now all natural numbers $k$ for which $\gamma_{r}(m, k) \neq 0$. From Lemma 11 we know that $\gamma_{r}(m, k)=0$ for $|v-z|>r$. Hence, we only need to consider the cases where $|v-z| \leq r$.

- $\quad v=\max (z-r, 0)$ : If $z-r \leq 0$, then this case does not occur; otherwise there is only one $k$ for which $\gamma_{r}(m, k) \neq 0$, and we obtain the summand $b^{-\mu_{r, \operatorname{per}}(m)}$.

- $\quad v=\max (z-r+1,0)$ : Again if $z-r+1 \leq 0$, then this case does not occur; otherwise we can bound this summand from above by $b^{-\mu_{r \text {,per }}(m)-1}$.

- $\max (z-r+1,0)<v \leq z$ : First, let $v=1$. Then $\kappa_{1}=\eta_{z}$ and $a_{1}=e_{z}$. Therefore, $k$ is fixed, $\mu_{r, \text { per }}(m, k)=\mu_{r, \text { per }}(m)$, and $b^{-\mu_{r, \text { per }}(m, k)}=b^{-\mu_{r, \text { per }}(m)}$. Let now $v>1$, which implies $z>1($ as $z \geq v)$ and $z-v+2 \leq r$. In this case

$$
\left(a_{2}, \ldots, a_{v}, \kappa_{2}, \ldots, \kappa_{v}\right)=\left(e_{z-v+2}, \ldots, e_{z}, \eta_{z-v+2}, \ldots, \eta_{z}\right) .
$$

Thus,

$$
\begin{aligned}
\mu_{r, \operatorname{per}}(m, k) & =\mu_{z-v+1, \operatorname{per}}(m)+a_{1}+\mu_{r-(z-v+2), \operatorname{per}}\left(k^{\prime}, k^{\prime}\right) \\
& \geq \mu_{r, \operatorname{per}}(m)+a_{1}-a_{v-z+r} .
\end{aligned}
$$

Note that $v-z+r>1$. Let $a_{v}^{\prime}=a_{1}-a_{v-z+r}>v-z+r-2$. Then the sum over all $k$ for which $1<v \leq z$ is bounded by

$$
b^{-\mu_{r, \mathrm{per}(m)}}(b-1) \sum_{v=2}^{z} \sum_{a^{\prime}=v-1}^{\infty} b^{-a^{\prime}} \leq b^{-\mu_{r, \mathrm{per}}(m)} \sum_{v=2}^{\infty} b^{-v+2} \leq b^{-\mu_{r, \mathrm{per}}(m)} \frac{b}{b-1} .
$$


- $\quad z+1 \leq v \leq z+r-2$ : If $z=1$, then $2 \leq v \leq r-1$ and, by Lemma 11, we have $\eta_{1}=\kappa_{v}$ and $e_{1}=a_{v}$. In this case $\mu_{r, \text { per }}(m, k)=\mu_{r, \text { per }}(k)$ and

$$
\begin{aligned}
\mu_{r, \mathrm{per}}(k)-\mu_{r, \mathrm{per}}(m) & =\left(a_{1}-a_{v}\right)+\cdots+\left(a_{v}-a_{v}\right)+(r-v)\left(a_{v}-a_{v}\right) \\
& =a_{1}^{\prime}+\cdots+a_{v-1}^{\prime},
\end{aligned}
$$

where $a_{i}^{\prime}=a_{i}-a_{v}$ and $a_{1}^{\prime}>\cdots>a_{v-1}^{\prime}>0$. The sum over all $k$ for which $2 \leq v \leq r-1$, and $\gamma_{r}(m, k) \neq 0$, is then bounded by

$$
\begin{aligned}
& \sum_{v=2}^{r-1}(b-1)^{v-1} \sum_{a_{1}>\cdots>a_{v-1}>a_{v}=e_{1}>0} b^{-\mu_{r, \mathrm{per}}(k)} \\
& \leq b^{-\mu_{r, \mathrm{per}}(m)} \sum_{v=2}^{r-1}(b-1)^{v-1} \sum_{a_{1}^{\prime}>\cdots>a_{v-1}^{\prime}>0} b^{-a_{1}^{\prime}-\cdots-a_{v-1}^{\prime}} \\
& \quad \leq b^{-\mu_{r, \operatorname{per}}(m)} \sum_{v=2}^{r-1} b^{-(v-2)} \\
& \leq b^{-\mu_{r, \operatorname{per}(m)}} \frac{b}{b-1} .
\end{aligned}
$$

For $z>1$ and $z+1 \leq v \leq z+r-2$ we have

$$
\left(a_{v-z+2}, \ldots, a_{v}, \kappa_{v-z+2}, \ldots, \kappa_{v}\right)=\left(e_{2}, \ldots, e_{z}, \eta_{2}, \ldots, \eta_{z}\right)
$$

and $v-z+2 \leq r$. Thus,

$$
\begin{aligned}
\mu_{r, \operatorname{per}}(m, k)= & a_{1}+\cdots+a_{v-z+1}+e_{1}+\mu_{r-(v-z+2), \operatorname{per}}\left(m^{\prime}, m^{\prime}\right) \\
\geq & \mu_{r, \operatorname{per}}(m)-\mu_{r-1, \operatorname{per}}\left(m^{\prime}\right)+a_{1}+\cdots+a_{v-z+1} \\
& \quad+\mu_{r-(v-z+2), \operatorname{per}}\left(m^{\prime}, m^{\prime}\right) \\
\geq & \mu_{r, \operatorname{per}}(m)+a_{1}^{\prime}+\cdots+a_{v-z+1}^{\prime},
\end{aligned}
$$

where $a_{i}^{\prime}=a_{i}-e_{2}=a_{i}-a_{v-z+2}$ and $a_{1}^{\prime}>\cdots>a_{v-z+1}^{\prime}>0$. Thus, the sum over all $k$ for which $z+1 \leq v \leq z+r-2$ and $\gamma_{r}(m, k) \neq 0$ is bounded by

$$
\begin{aligned}
& b^{-\mu_{r, \mathrm{per}}(m)} \sum_{v=z+1}^{z+r-2}(b-1)^{v-z+1} \sum_{a_{1}^{\prime}>\cdots>a_{v-z+1}^{\prime}>0} b^{-a_{1}^{\prime}-\cdots-a_{v-z+1}^{\prime}} \\
& \leq b^{-\mu_{r, \mathrm{per}}(m)} \sum_{v=z+1}^{z+r-2} b^{-1-\cdots-(v-z)} \\
& \leq \frac{b^{-\mu_{r, \mathrm{per}}(m)}}{b-1}
\end{aligned}
$$


- $v=z+r$ : In this case $\mu_{r, \text { per }}(m, k)=a_{1}+\cdots+a_{r}-\mu_{r, \text { per }}(m)+\mu_{r, \text { per }}(m)$, where $\mu_{r, \operatorname{per}}(m) \leq r a_{r+1}$. Thus,

$$
a_{1}+\cdots+a_{r}-\mu_{r, \text { per }}(m) \geq\left(a_{1}-a_{r+1}\right)+\cdots+\left(a_{r}-a_{r+1}\right)
$$

and $a_{1}>\cdots>a_{r}>a_{r+1}$. Hence, the sum over all $k$ for which $v=z+r$ is bounded by

$$
(b-1)^{r} b^{-\mu_{r, \mathrm{per}}(m)} \sum_{a_{1}>\cdots>a_{r}>0} b^{-a_{1}-\cdots-a_{r}} \leq b^{-\mu_{r, \mathrm{per}}(m)} b^{-r(r-1) / 2} .
$$

- $v=z+r-1$ : $\quad$ in this case $\mu_{r, \text { per }}(m, k)=a_{1}+\cdots+a_{r}-\mu_{r, p e r}(m)+$ $\mu_{r, \text { per }}(m)$, where now $a_{r}=e_{1}$ and $\kappa_{r}=\eta_{1}$ are fixed. Hence, the sum over all $k$ for which $v=z+r-1$ is bounded by

$$
(b-1)^{r-1} b^{-\mu_{r, \operatorname{per}}(m)} \sum_{a_{1}>\cdots>a_{r-1}>0} b^{-a_{1}-\cdots-a_{r-1}} \leq b^{-\mu_{r, \operatorname{per}}(m)} b^{-(r-1)(r-2) / 2} .
$$

By summing up the bounds obtained for each case, we obtain the result.

This implies the following theorem.

THEOREM 14. Let $r>1$. Then for any $k \in \mathbb{N}$ we have

$$
\begin{aligned}
|\hat{f}(k)| \leq \sum_{s=v}^{r} \mid & \int_{0}^{1} f^{(s)}(x) d x \mid \frac{b^{-\mu_{s, \mathrm{per}}(k)}}{(2 \sin (\pi / b))^{s}}\left(1+\frac{1}{b}+\frac{1}{b(b+1)}\right)^{\max (0, s-2)} \\
& +\int_{0}^{1}\left|f^{(r)}(x)\right| d x \frac{2 b^{-\mu_{r, \mathrm{per}}(k)}}{(2 \sin (\pi / b))^{r}}\left(1+\frac{1}{b}+\frac{1}{b(b+1)}\right)^{r-2} \\
& \times\left(3+\frac{2}{b}+\frac{2 b+1}{b-1}\right)
\end{aligned}
$$

for all $f \in \mathcal{H}_{r}$, where for $v>r$ the empty sum $\sum_{s=v}^{r}$ is defined as 0 .

REMARK 15. This theorem can easily be generalized to tensor product spaces, for which the reproducing kernel is just the product of the one-dimensional kernels.

\section{On the Walsh coefficients of smooth periodic functions}

We consider a subset of the previous reproducing kernel Hilbert space, namely, let $\mathcal{H}_{r \text {,per }}$ be the space of all functions $f \in \mathcal{H}_{r}$ which satisfy the condition $\int_{0}^{1} f^{(s)}(x) d x=0$ for $0 \leq s<r$. This space also has a reproducing kernel, which is given by

$$
\mathcal{K}_{r, \operatorname{per}}(x, y)=(-1)^{r+1} \frac{\widetilde{B}_{2 r}(x-y)}{(2 r) !}=(-1)^{r+1} \widetilde{b}_{2 r}(x-y) .
$$


The inner product is given by

$$
\langle f, g\rangle_{r, \text { per }}=\int_{0}^{1} f^{(r)}(x) g^{(r)}(x) d x .
$$

We also have the representation

$$
f(y)=(-1)^{r+1} \int_{0}^{1} f^{(r)}(x) \widetilde{b}_{r}(x-y) d x .
$$

For this space we can obtain an analogue to Theorem 14.

THEOREM 16. Let $r>1$. Then, for any $k \in \mathbb{N}$, we have

$$
\begin{aligned}
|\hat{f}(k)| \leq \int_{0}^{1} & \left|f^{(r)}(x)\right| d x \\
& \times \frac{2 b^{-\mu_{r, \operatorname{per}(k)}}}{(2 \sin (\pi / b))^{r}}\left(1+\frac{1}{b}+\frac{1}{b(b+1)}\right)^{r-2}\left(\frac{7}{2}+\frac{2}{b}+\frac{2 b+1}{b-1}\right),
\end{aligned}
$$

for all $f \in \mathcal{H}_{r, \text { per }}$.

REMARK 17. This theorem can easily be generalized to tensor product spaces, for which the reproducing kernel is just the product of the one-dimensional kernel.

REMARK 18. For $2 \leq b \leq 4$ we have

$$
\left(2 \sin \frac{\pi}{b}\right)^{-1}\left(1+\frac{1}{b}+\frac{1}{b(b+1)}\right)<1,
$$

and so, for these cases, the constants in the theorems above decrease as $v, r$ increase.

REMARK 19. Because the Walsh coefficients considered in this paper converge quickly, the Walsh series for functions $f$ with smoothness $r>1$ converges absolutely (we have $\sum_{k=0}^{\infty} b^{-\mu_{r, \text { per }}(k)} \leq \sum_{k=0}^{\infty} b^{-\mu_{r}(k)}<\infty$ for $r>1$ and $\sum_{k=0}^{\infty} b^{-a_{1}-\lambda a_{2}}<\infty$ for $\lambda>0$ ) and we have (see [4])

$$
f(x)=\sum_{k=0}^{\infty} \hat{f}(k) \mathrm{wal}_{k}(x) \quad \text { for } 0 \leq x<1 .
$$

\section{Lower bounds}

Fine [6, Theorem VIII] proved that the only absolutely continuous functions whose Walsh coefficients decay faster than $1 / k$ are the constants.

This result can be extended in the following way. Let $f$ have smoothness $r>1$ (that is, the $\lfloor r\rfloor$ th derivative has at least bounded variation of order $r-\lfloor r\rfloor$ ). Let $k^{(1)}=k^{\prime}, k^{(2)}=k^{\prime \prime}$ and $k^{(s)}=k^{(s-1)}-\kappa_{s} b^{a_{s}-1}=\kappa_{s+1} b^{a_{s+1}-1}+\cdots+\kappa_{v} b^{a_{v}-1}$ for $1 \leq s<v$ and let $k^{(v)}=0$. 
Using $\int_{0}^{1} f(x) \overline{\operatorname{wal}_{k}(x)} d x=-\int_{0}^{1} f^{\prime}(x) J_{k}(x) d x$ and Lemma 1

$$
\begin{array}{r}
\hat{f}(k)=-b^{-a_{1}}\left(1-\omega_{b}^{-\kappa_{1}}\right)^{-1} \hat{f}^{\prime}\left(k^{\prime}\right)-b^{-a_{1}}\left(1 / 2+\left(\omega_{b}^{-\kappa_{1}}\right)^{-1}\right) \hat{f}^{\prime}(k) \\
-b^{-a_{1}} \sum_{c=1}^{\infty} \sum_{\vartheta=1}^{b-1} b^{-c}\left(\omega_{b}^{\vartheta}-1\right)^{-1} \hat{f}^{\prime}\left(\vartheta b^{a_{1}+c-1}+k\right),
\end{array}
$$

where $\hat{f}^{\prime}(k)$ denotes the $k$ th Walsh coefficient of $f^{\prime}$.

Using Lemma 7 we obtain for fixed $k^{(1)}$ and $\kappa_{1}$ that

$$
\lim _{a_{1} \rightarrow \infty} b^{a_{1}} \hat{f}(k)=-\left(1-\omega_{b}^{-\kappa_{1}}\right)^{-1} \hat{f}^{\prime}\left(k^{(1)}\right) .
$$

Applying this result inductively we obtain for $s \leq \min (\lfloor r\rfloor, v)$ and fixed $k^{(s)}$ and $\kappa_{1}, \ldots, \kappa_{s}$ that

$$
\begin{aligned}
\lim _{a_{s} \rightarrow \infty} & b^{a_{s}} \ldots \lim _{a_{2} \rightarrow \infty} b^{a_{2}} \lim _{a_{1} \rightarrow \infty} b^{a_{1}} \hat{f}(k) \\
= & -\lim _{a_{s} \rightarrow \infty} b^{a_{s}} \ldots \lim _{a_{2} \rightarrow \infty} b^{a_{2}}\left(1-\omega_{b}^{-\kappa_{1}}\right)^{-1} \hat{f}^{\prime}\left(k^{(1)}\right) \\
= & (-1)^{s} \prod_{s^{\prime}=1}^{s}\left(1-\omega_{b}^{-\kappa_{s^{\prime}}}\right)^{-1} \hat{f^{(s)}}\left(k^{(s)}\right) .
\end{aligned}
$$

This implies that if $\hat{f}(k)$ decays faster than $b^{-a_{1}-\cdots-a_{s}}$ for all $k=\kappa_{1} b^{a_{1}-1}+\cdots+$ $\kappa_{v} b^{a_{v}-1}$ with $0<\kappa_{1}, \ldots, \kappa_{v}<b$ and $v \geq s$, then $\hat{f^{(s)}}(k)=0$ for all $k$ and, therefore, $f^{(s)}=0$, that is, $f$ is a polynomial of degree at most $s-1$.

THEOREM 20. Let $f$ have smoothness $r>1$. Then iffor some $1 \leq s \leq r, \hat{f}(k)$ decays faster than $b^{-a_{1}-\cdots-a_{s}}$ for all $k=\kappa_{1} b^{a_{1}-1}+\cdots+\kappa_{v} b^{a_{v}-1}$ with $0<\kappa_{1}, \ldots, \kappa_{v}<b$ and $v \geq s$, that is,

$$
\lim _{a_{s} \rightarrow \infty} b^{a_{s}} \cdots \lim _{a_{2} \rightarrow \infty} b^{a_{2}} \lim _{a_{1} \rightarrow \infty} b^{a_{1}} \hat{f}(k)=0 \quad \text { for all } k \text { with } v \geq s,
$$

then $f$ is a polynomial of degree at most $s-1$.

\section{References}

[1] N. Aronszajn, 'Theory of reproducing kernels', Trans. Amer. Math. Soc. 68 (1950), 337-404.

[2] H. E. Chrestenson, 'A class of generalised Walsh functions', Pacific J. Math. 5 (1955), 17-31.

[3] J. Dick, 'Explicit constructions of quasi-Monte Carlo rules for the numerical integration of high dimensional periodic functions', SIAM J. Numer. Anal. 45 (2007), 2141-2176.

[4] - 'Walsh spaces containing smooth functions and quasi-Monte Carlo rules of arbitrary high order', SIAM J. Numer. Anal. 46 (2008), 1519-1553.

[5] J. Dick and F. Pillichshammer, 'Multivariate integration in weighted Hilbert spaces based on Walsh functions and weighted Sobolev spaces', J. Complexity 21 (2005), 149-195.

[6] N. J. Fine, 'On the Walsh functions', Trans. Amer. Math. Soc. 65 (1949), 372-414.

[7] K. Niederdrenk, Die endliche Fourier- und Walshtransformation mit einer Einführung in die Bildverarbeitung (Vieweg, Braunschweig, 1982). 
[8] G. Pirsic, 'Embedding theorems and numerical integration of Walsh series over groups', PhD Thesis, University of Salzburg, 1997.

[9] W. R. Wade, 'Recent developments in the theory of Walsh series', Internat. J. Math. Math. Sci. 5 (1982), 625-673.

[10] G. Wahba, Spline Models for Observational Data, CBMS-NSF Regional Conference Series in Applied Mathematics, 59 (Society for Industrial and Applied Mathematics (SIAM), Philadelphia, PA, 1990).

[11] J. L. Walsh, 'A closed set of normal orthogonal functions', Amer. J. Math. 55 (1923), 5-24.

JOSEF DICK, School of Mathematics and Statistics, University of New South Wales, Sydney 2052, Australia

e-mail: josef.dick@unsw.edu.au 